\section{USO DA CINZA INSOLUVEL EM ACIDO PARA DETERMINAR O CONSUMO DE FORRAGEM DOS BOVINOS *}

\author{
CARMEN NEUSA MARTINS CORTADA \\ Médica Veterinária \\ LICIO VELLDSO \\ Professor Titular \\ Faculdade de Medicina Veterinaria \\ e Zootecnia da USP
}

\section{INTRODULAO}

o consumo de matéria seca é um importante fator dentre aqueles relacionados com - valor nutritivo do pasto tropical. D ideal seria que ele fosse determinado com o animal no pasto, onde a habilidade deste em selecionar a parte mais nutritiva ou o "instinto seletivo". possa ser mais livremente manifestada. No entanto, os métodos disponiveis para medir o consumo em condicốs adequadas estzo sujeitos a grandes erros. D consumo pode tambem ser medido com emprego de um indicador interno, conhecendo-se as concentracores deste nas fezes e na forragem, alem do total de materia seca excretada.

D objetivo deste trabalho foi comparar - consumo obtido com o emprego da cinza insolúvel em acido (CIA), que é um marcador interno, com o observado diretamente nos animais mantidos em gaiulas de metabolismo. As cinzas insoluveis em acido foram determinadas por dois processos anallticos. empreganda $\mathrm{HCl}$ concentrado e HCl $2 \mathrm{~N}$.

\section{REUISAO DA LITERATURA}

A cinza insoluvel em acido HCl concentrado foi empregada para estimar consumo de forragem, de ovinos mantidos em condicồes de pasto por SHRIVASTAUA \& TALAPATRA, 10, 1962, com resultados considerados 5 atisfalorios pelos autores. Neste trabalho a exerecaro fecal foi medida atraves do uso ó bolsas coletoras de fezes.

No Brasil, ANDREASI et ali 1,2 (1960/62, 1963/64) haviam aplicado a método dos indicadores com oxido cromico e cromogenios, para a determinacăo da digestibilidade aparente e para avaliacarodo consumo de materia seca por Bos indicus. KULRIUUES, 5, em ï75, utii:zou a cinza insolúvel em acido HCl $2 \mathrm{~N}$ para estimar consumo de materia seca. 0 autor trabathou com ovinos e associou o oxido cromico com a cinza insolúvel em acido para estimar a excrecazo fecal. Ds resultados foram considerados tambem satisfatorios. Para determinar consumo de forragem ja foram testados varios outros indicadores como o eromogenis, a lignocelulose, a ligninde a sllica (PEZD, et alii, 8, 1977 e DYNE \& MEYER， 5, 1964)

MATERIAL E METODOS

Foram utilizados seis bovinos mesticos da raca holandesa preta e branca, machos inteiros, com peso vivo medio inicial de $195,600 \mathrm{~kg}$ e idade variando entre 16 e 33 meses. a alimento se contituiu em feno de Cynodon dactylon hlorido Coasteross-1, fornecido picado e a vontade.

o experimento constou de um periodo preliminar de 16 dias, para que os animais se adaptassem ao alimento, às condiços da gaiola e às condicôes ambientais. Os animais perinaneceram en gaiolas de metabolismo
Resumo da Dissertação de Mestrado apresentada à Faculdade de Medicina Veterinária e Zootecnia da USP. 
contruldas de madejia, tendo suas fezes recolhidas em caixa tambem de madejra. recoberta com lona plastica: 0 perlodo experimental teve a duracaro dé sele dias. Diarjamente reculhiam-se as fezes e verificava-se o consumo de feno.

Nas amostras secas a moldas de feno e de fezes loram determinados os teores de materia seca, atraves dos metodos analliticos recomendados Delo ASSOCIATION OF OFFICIAL ANALYTICRL CHEMISTS, 3 (1970) e a energia bruta foi determinada em bomba colorimetria tipo pare, sendo as cinzas insoluveis em bcido determinadas segundo KEULEN \& YDUNG, 7 (1977).

- consumo de matéria seca pelo metodo do indicador foi calculado sequndo a lormula seguinte, usada por CRAMPTDN \& HARRIS. 4 (1969) \& Dor SHRIUASTAUA \& TALAPATRA.. 10 (1962).

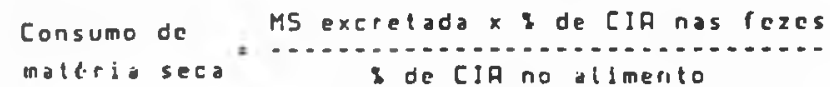

- detineamento experimental empregado foi o inteiramente casualizado..

Comparou-se o consumo de materia seca em g/kg 0,75 determinado pelos métodos da cinza insoluvel em acido HCl concentrado, da cinza insoluvel em acido HCl $2 \mathrm{~N}$ e pelo consumo observado diretamente no experimento. Foram analisadas estitisticamente as medias obtidas com os seis animais, durante os sete dias de collheita.

A andlise estatlstica compararam-se as medias de consumo de materia seca, obtidas pélos tres métodos atraves da analise de variancia e da correlaça simples (GOMES, 6, 1981)

\section{RESULTADOS E DISCUSSAD}

D consumo medio de materia seca
g/kg0,75 ldia estimado pelo metodo da
cinza insoldvel em HCl $2 N$ foi maior que o
estimado pelo método da cinza insoldvel em
HCl concentrado e tambem major que o con-
sumo observado, mas a diferenca existente
entre as medias nao foi estatisticamente
significativa (Tab. 1)

TABela 1 - Consumo médio de matéria seca $\mathrm{g} / \mathrm{kg}^{0,75} / \mathrm{dia}$ obtido com - emprego dos ties métodos

\begin{tabular}{|c|c|c|c|c|}
\hline & $\begin{array}{c}\text { Consuto } \\
\text { Direto } \\
\text { (observado) }\end{array}$ & $\begin{array}{c}\text { Cin } \\
\text { HCI Corcen- } \\
\text { baco }\end{array}$ & $\mathrm{H} \in \mathrm{CIAS}$ 2R: & $\underset{F}{\text { Meste }}$ \\
\hline $\begin{array}{l}\text { liútero de } \\
\text { Observaçōes }\end{array}$ & 6 & 6 & 6 & \\
\hline $\begin{array}{l}\text { Nédia } \neq s \\
s(\dot{m}) \\
C . v . \%\end{array}$ & $\begin{array}{c}60,75-7,79 \\
3,18 \\
21,16\end{array}$ & $\begin{array}{c}72,45 \pm 20,26 \\
4, \pm 9 \\
24,36\end{array}$ & $\begin{array}{c}77.78 \pm 10,25 \\
4,43 \\
13.95\end{array}$ & $\pi . \Xi$. \\
\hline
\end{tabular}

$\mathrm{ClA}=$ cinza insolúvel em ácido

$\mathrm{CV}=$ coeficiente de variạ̧āo

$\mathrm{HCL}=$ ácido eloridrico

$S(\hat{m})=$ erro padrão da média

ns $=$ não significativo ao nivel de $5 \%$ de probabilidade 
Este resultado concorda com aqueles obtidos Dor SHRIURSTAUA \& TALAPATRA, 10 (1962) e por RODRIGUES, 9 (1979)
A correlas a simples entre os valoces de consumo de materia seca g/kg0,75/dia foi estatisticamente significativa $(P, 0,05)$ $(T a b .2)$ TABELA 2 - Coeficiente de correlacao $5 i$ mojes enire as medias de
consumo ce materia secaglkgo, observadas atraves
dos tres metodos de determinacjo.

\begin{tabular}{|c|c|c|c|c|}
\hline$x$ & & $y$ & & ${ }^{r}(x y)$ \\
\hline \multirow[t]{2}{*}{$\mathrm{cl}: \mathrm{s} / \mathrm{kE}^{0,75}$} & 2 & $\mathrm{CAs} / \mathrm{kg}^{0.75}$ & 2 & $0,955^{*}$ \\
\hline & & $\mathrm{C}: \mathrm{S} / \mathrm{k} \cdot \mathrm{E}^{0,75}$ & 3 & $0.959^{\circ}$ \\
\hline $\mathrm{CN}:=/ \mathrm{kE}^{0,75}$ & 2 & $\mathrm{crss} / \mathrm{kg}^{0.75}$ & 3 & 0.997 \\
\hline
\end{tabular}

$\mathrm{CMS} / \mathrm{KC}^{0,75}$ - consumo de mattria seca/ $/ \mathrm{kg}^{0.75}$

- significativo ao nivel de $5 \%$ de probabilidade

1 - observacto direta durante o experimento

2 - CLA HCl concentrado

3 - CLA HCl 2N
As altas correlacoes, constantes da Tab.2, de certa forma confirmam a validade do uso dos metodos de CIA em HCl concentrado e $\mathrm{HCl} 2 \mathrm{~N}$, para estimativa do consumo de materia seca pelos bovinos. 5ao recomendaveis estudos posteriores com outras for ragens tropicais e com o animal mantido em regime de pastejo, visto que a maior parte das volumosos e consumida nestas conoicónes.

\section{CONCLUSAO}

O emprego da cinza insoluvel em acido, permitiu estimar convenientemente o consumo medio de materia seca g/kg0,75/cia, como constatado pelos elevados coeficientes de corre! acto entre os metodos.
CORTRDA, C.N.M.: VELLOSO, L. Aeid insoluble ash used to determine dry matter intake by cattle. Rev.Fac.Med.Vet. Zootec. Univ.5.Paulo, 24(9): 61-64, 1987 .

SUMMARY: Six young crossbred bulls vere used to determine dry matter intake by cattle fed a tropical grass hay (Cynodon dactylon, Coasteross 1). Digestion trial lasted for 23 days being the last 7 days for feed and feces sample coliections. Dry matie: intake was cal a a aled by use of adequate formulas and incicator methoo. Dry matter intake was calculated by acid insoluble ash $H C L 2 N$ and concentrated $H C L$ and also by direct consumption. ihere was no statistical difference among methoos for dry matler consumption.

UNITSRMS: Indicators, acid insoluble ast. Forage, dry matter intake; Nutrition of eattle

REFERENCIAS BIBLIOGRAFICAS

9 - ANDReasi, F.; masotTl, N.; Veiga, J.S.M. Rplicaca dos métodos dos indicadores - bxido cromico e cromogenios - Dara determinaca da digestibilidade aparente. em zebu (Bos indicus). Rev.Fac. Med.vet. Zootec.Univ.5.Paulo, 6(4):401-433. 1962 .
2 - ANDREASI, F.; MASOTTI, N.; VEIGA, J.S.M. Avaliaga do consumo de forragens e determinacaro da excrefäo de fezes ein Zebu (Bos indicus). atraves dos indicadores oxido e eromogenios. Rev. Fac. Med.vet. Zootec. Univ.5.Paulo, 7(1):154-189,1963/64.

Rev.Fac.Med.Vet.Zootec.Univ.S.Poul. . . f(1):61-64, 1987. 
3 - ASSOCIATION OF THE OFFICIAL ANALYTICAL CHEMISTS. 11.ed. Washington, 1970.

4 CRAMPTON, E.W. \& HARRIS, L.E. Applied
animal nutrition. 2.ed. San Francisco, W.H. Freeman, 1969.

5 - DYNE, G.M. van \& MEYER, J.H. Forage intake by cattle and sheep on dry annual range. J.anim.Sci., 23:11081115,1964 .

6 - Gomes, F.P. Curso de estatistica experimental. 9.ed. Piracicaba, Nobel, 1981.

7 - KEULEN. J. van \& YOUNG, B.A. Evaluation of acid-insoluble ash as a natural marker in ruminant digestibility studies. J.anim.Sci.. $44: 282-287,1977$.

8 - PEZO, D.; VOHNOUT, K.; VELARDE, C.L.; CAMARGo, A. Prediccion de consumo de pastos em laboratorio. Turrialba, 27:157-162, 1377 .

9 - RODRIgues, A.R. Determinacão da digestibilidade e consumo de gramlneas e leguminosas, em ovinos, pelo oxido cromico e cinzas insolúveis em acido. Belo Horizonte, 1979. ITese de Escola de Veterinaria da Universidade Federal de Minas Geraisl

10 - SHRivastava, U.5. \& TALAPATRA, 5.K. Pasture studies in Uttar Pradesh. 1II. Determination of pasture consumption by grazing animal. Indian J. Diary Sci., 15:151-166, 1962 . 